\title{
The effects of mergers and acquisitions on the firm size distribution
}

\author{
Elena Cefis • Orietta Marsili • Hans Schenk
}

Published online: 12 June 2008

(C) The Author(s) 2008

\begin{abstract}
This paper provides new empirical evidence on the effects of mergers and acquisitions (M\&As) on the shape of the firm size distribution, by using data of the population of manufacturing firms in the Netherlands. Our analysis shows that M\&As do not affect the size distribution when we consider the entire population of firms. When we focus on the firms involved in an M\&A event, we observe a shift of the firm size distribution towards larger sizes. Firm size distribution becomes more concentrated around the mean, less skewed to the right hand side, and thinner at the tails as a whole. The shift toward higher sizes due to M\&A is not uniform but affects firms of different sizes in different ways. While the number of firms in the lower tail decreased, the number of firms in the central size classes increased substantially and outweighed the increase in the number (and mean size) of firms in the upper tail of the distribution (consequently the overall market concentration measured by the Herfindahl index declines). M\&As lead to a departure from log-normality of the firm size distribution, suggesting that external growth does not follow Gibrat's law. Our counterfactual analysis highlights that only internal growth does not affect the shape of the size distribution of firms. On the contrary, it
\end{abstract}

\footnotetext{
E. Cefis $(\varangle)$

University of Bergamo and T.C. Koopmans Research Institute, Utrecht University, Janskerkhof 12, 3512 BL, Utrecht, The Netherlands

e-mail: e.cefis@econ.uu.nl

O. Marsili

Rotterdam School of Management, Erasmus University,

Rotterdam, The Netherlands

e-mail: omarsili@rsm.nl

H. Schenk

Utrecht School of Economics, Utrecht University, Utrecht, The Netherlands

e-mail: hans.schenk@econ.uu.nl
} 
suggests that the change in the size distribution is almost entirely due to the external growth of the firms.

Keywords Firm size distribution • Mergers and acquisitions •

Firm entry and exit • Industry concentration

JEL Classification $\mathrm{L} 11 \cdot \mathrm{L} 25 \cdot \mathrm{D} 21 \cdot \mathrm{C} 14$

\section{Introduction}

Empirical studies have repeatedly shown that firm size distributions in industrial countries are highly skewed, or, in other words, that a small number of large firms coexist with a large number of small firms. The firm size distribution within an industry indicates the degree of industrial concentration and therefore is of particular interest for antitrust policy.

Starting with Gibrat (1931), firm size has often been described by lognormal distributions. Indeed, this distribution has been interpreted as the outcome of the "Law of Proportionate Effects", which predicts that firm size follows a random walk and, hence, that the growth of firms is erratic and independent of size. (For a survey see Sutton 1997; and Bottazzi et al. 2002) Gibrat's Law was originally used as an explanation of the highly skewed distribution of firm size and it has become, both empirically and theoretically, a benchmark for discussing the processes of firm growth (McCloughan 1995; Geroski 1999; Lotti and Santarelli 2004; Cefis et al. 2007). Recently, Luttmer (2007) and Bottazzi and Secchi (2006) have proposed different theoretical models that, deviating from Gibrat's Law, are able to explain internal firm growth giving, as an outcome, size distributions that are consistent with salient features of the observed firm size distributions.

The upper tail of these highly skewed size distributions has often been described by the Yule or Pareto (also known as "Power Law") distributions. If a discrete random variable, such as firm size, is Pareto-distributed, it means that the frequency of the variable above a certain threshold is inversely proportional to the value of the variable. In the case of firm size, the Power Law predicts that the frequency of firms above a certain size (or a minimum size) is inversely proportional to firm size.

Several studies in industrial economics have empirically tested whether firm size is Pareto-distributed and have formulated models able to generate Paretolike distributions (e.g. Ijiri and Simon 1974). If firm size is distributed according to a Pareto distribution, the coefficient of the distribution (the slope of the Pareto curve) is a measure of the degree to which business is concentrated in the larger firms in an industry or an economy, expressing the percentage of observations in the upper tail of the distribution. Furthermore, if Pareto distributed and with slope parameter within a certain range, the industrial structure would depend only on the interaction between firms and not on external factors or individual firm behaviour (Krugman 1996) 
Despite the vast literature that has investigated the firm size distributionsome of which will be discussed below-little attention has been devoted to the underlying form of firm growth, and in particular to the effects of internal firm expansion and of external firm growth.

The external firm growth, due to mergers and acquisitions (M\&As), may be of significance if the incidence (and/or size) of M\&As were not neutral with respect to the firm size. For example, suppose that merger-initiating firms (i.e. acquiring firms) are more ubiquitous in larger size classes. If such mergers are more effective in gaining size than would be the case with growth by internal expansion (which, in the short run, is obviously true) and sustainable (in the sense that they do not have to be broken up soon after initiation because of failures), then-ceteris paribus-we would expect a systematically higher growth rate in the upper tails of the size distribution. Growth, in other words, would then be dependent on firm size.

Also, it is important to note that the variance of a distribution is a measure of inequality, not concentration (Hannah and Kay 1981). Thus, trends in inequality and concentration will be similar if the number of firms remains constant, but when this latter number declines, concentration will increase while-depending on the size of acquired firms-the degree of inequality may rise as well as fall. Since mergers necessarily imply such a decline, concentration through merger may or may not increase inequality, whereas inequality will increase when concentration increases through internal growth.

That this concerns non-trivial questions is shown by the dramatic rise in M\&As during the second half of the 1990s (see Fig. 1). In fact, this so-called fifth merger wave exceeded everything that went on before, both in numbers and in deal value. During 1996-2000, more than $\$ 12,000$ billion was spent on mergers, $\$ 9,000$ billion of which by firms from Europe and the USA.

Among large firms, merger is also the most important cause of disappearance, especially in Europe and the USA (see Table 1). Among the US and the European Union's (EU's) 100 largest manufacturing firms, 30 were absorbed into another member of this size group within a 15-year period. In total, $15 \%$ of large firms disappeared through merger, except in Japan where large

Fig. 1 M\&As by total transaction value (trn US\$). Source: Schenk (2003)

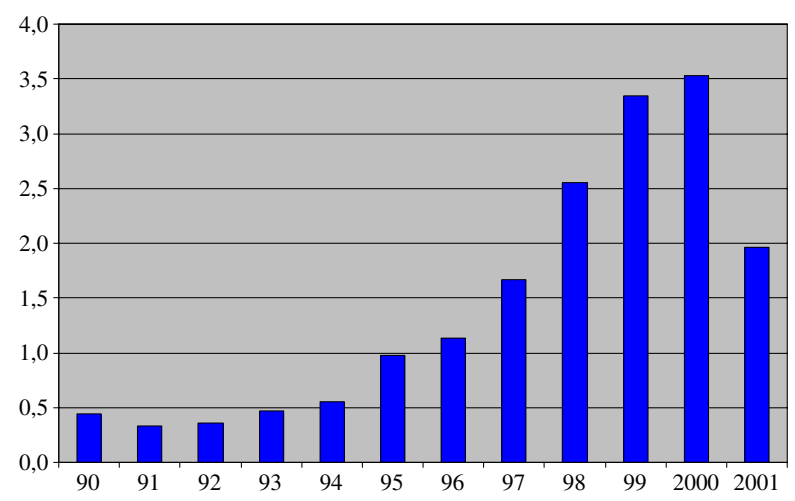


Table 1 Disposition of the Triad's largest manufacturers of 1978 with respect to 1993

\begin{tabular}{|c|c|c|c|}
\hline & USA & EU & Japan \\
\hline Liquidated & 0 & 1 & 0 \\
\hline Demerged & 0 & 2 & 0 \\
\hline Surpassed $^{\mathrm{a}}$ & 21 & 21 & 18 \\
\hline Survived & 58 & 57 & 78 \\
\hline Acquired(remaining in top-100) & 14 & 16 & 4 \\
\hline Other ${ }^{b}$ & 7 & 3 & 0 \\
\hline Total & 100 & 100 & 100 \\
\hline
\end{tabular}

In percentages; with respect to top-100 firms of each respective region. Note that the period does not cover the massive merger movement in the second half of the 1990s.

${ }^{a}$ Firms that dropped out of the top-100; no further information as to their disposition available

${ }^{b}$ Firms that dropped out of the top-100 because of reclassification to another sector (mainly services)

Source: Schenk (1997)

firms apparently are much less active in mergers. Evidently, extending the population to smaller size categories will increase the percentage of merger disappearances. For example, of the 3,011 firms quoted on the London Stock Exchange in 1950, no less than 1,265 (or 42\%) appear to have been taken over by 1977 (Odagiri 1992).

The purpose of the paper is, therefore, to analyze the effects of M\&As on the firm size distribution and on concentration proxies. The aim is to supply new empirical evidence on a topic scarcely investigated. The research is meant to be a first step in the analysis of the firm's growth process when considering not merely internal growth (i.e. growth due to the internal capacities to expand in terms of total sales or number of employees) but also external growth (i.e. growth due to M\&As).

In Section 2, we discuss some crucial results previously obtained in the literature. Section 3 describes the database and the variables under scrutiny. In Section 4, we examine the evidence on the aggregate size distributions, while in Section 5, we consider only the size distribution of firms involved in a merger and/or an acquisition. Section 6 discusses the results of the counterfactual analysis and Section 7 concludes.

\section{Literature review and discussion}

The increasing importance of large firms is hardly contested. Indeed, the data throughout the previous century, perhaps excluding the 1930s through to the 1950s, show increasing aggregate concentration in manufacturing, mining and distribution for most European countries well into the 1990s, the Netherlands included (Schenk 1997). Data on the U.S. reveals an undulating pattern of aggregate concentration until the early 1990s, after which it rises sharply (Pryor 2001). Such increasing dominance can result from stochastic processes, or can be the inevitable result of modern technology and large-scale production. In all cases, however, it can be carried by M\&As. 
Ijiri and Simon (1971) was the first of only a small number of empirical studies focusing on the effects of M\&As on the firm size distribution. ${ }^{1}$ Comparing firm size distributions in 1956 and in 1957 in a sample of large American firms, Ijiri and Simon tested the effects of M\&As on the concentration measure $\beta$ (the slope of the Pareto curve). Their results suggested that M\&As do not greatly affect $\beta$, a conclusion that has long remained a prime source of reference no matter how counterintuitive. Furthermore, Ijiri and Simon proposed that, during the 1950s and 1960s, the size distribution of the 500 largest firms remained relatively unchanged. Any growth of the firms in question then takes the form of a parallel upward shift in the (partial) firm size distribution, the degree of shift depending on the growth rate that is applicable to all firms in the relevant population, regardless of size. Their analysis thus supports the proposition that firm growth due to M\&As would follow Gibrat's Law to the same extent as would internal growth. ${ }^{2}$

However, these results may have been biased because the mid-1950s witness an exceptionally low merger incidence. Thus, comparing the firm size distribution of 1957 with that of 1956 would not lead one to expect to find significant differences, if any. Similarly, and despite Ijiri and Simon's claims to the contrary, M\&As only appeared to catch on in the late 1960s (starting what would later become known as the third merger wave; see Blair 1972), so that it would not be surprising to find that the size distribution of the top-500 firms did not change much from the early 1950s through to the mid 1960s.

Indeed, in their later work, Ijiri and Simon (1974) modified their earlier conclusions. Studying the 831 largest industrial firms according to the annual Fortune rankings for 1969, they found a firm size distribution that departed significantly from the straight-line Pareto curve. Two potential explanations were subsequently investigated, one of which concerned the effect of M\&As. ${ }^{3}$ They first grouped the 831 firms into nine size classes, and then observed the number of firms that were involved in mergers during the 20 preceding years, classifying these by (a) the post-merger size of the combined firms; (b) the premerger size of acquiring firms; and (c) the pre-merger size of acquired firms. The estimated number of firms if mergers are eliminated, then, was given by calculating over the size classes the number of 1969-firms minus the numbers classified as (a), plus those classified as (b) and (c). It turned out that 1,002 firms would have existed in 1969 instead of the actually observed 831 firms, and that the rate of disappearance due to merger was not independent of the size class, in the sense that smaller firms had a higher chance of being absorbed. A similar exercise involving assets produced a series of merger-free asset data by size

\footnotetext{
${ }^{1}$ In this paper, we do not distinguish between merger, on the one hand, and acquisition or takeover, on the other. All three terms will be used interchangeably.

${ }^{2}$ Notice that Ijiri and Simon (1971), contrary to common parlance, defined internal growth as growth due to mergers and acquisitions and external growth as growth due to growth from sources outside the population.

${ }^{3}$ Ijiri and Simon (1974) also studied a model in which a firm with a history of recent growth had a better chance for further growth than a firm of the same size the growth of which had taken place in the distant past. It appeared plausible and also found empirical support.
} 
class. Growth by assets as a result of merger appeared to be dependent on size class as well, in the sense that, without mergers, the total of assets present in the smaller size classes would have been higher than actually observed, whereas in the larger size classes, it would have been smaller than actually observed. ${ }^{4}$ By plotting the actual data and the estimated data against the theoretical sizerank relationship (the Pareto curve), Ijiri and Simon were able to confirm that M\&As had contributed appreciably to increasing the concentration measure and the concavity of the firm size distribution, i.e. a departure from the Pareto expectation. It is worth noticing that by far most of the increases in size-growth disparities were observed during the last few years of the period studied, i.e. when the third merger wave caught momentum.

Following somewhat different methodologies, but essentially retaining the idea of composing hypothetical 'merger-free' populations for comparison, other researchers have come up with similar results. Singh (1975) found that, from the second quintile upwards, the probability of being acquired declines monotonically with size, moderately at first but more sharply once the top size classes are reached. Similarly, Aaronovitch and Sawyer (1975) reinforced the finding that, among large companies, size and the probability of acquisition are inversely related. Among the smallest of size classes, the disappearance rate due to merger over a 12 -year period was $40.4 \%$, after which it declined systematically with increasing size, ultimately reaching $21.1 \%$ and $26.3 \%$ in the highest size classes. Other things being equal, this suggests that smaller firms disappear as bigger firms acquire them, while some bigger firms disappear because they merge among themselves, thus creating a size-stronger segment in the upper tails of the distribution.

According to Hannah and Kay (1977), the effects of merger on growth are so strong, that without mergers, smaller firms would have grown faster than larger firms. ${ }^{5}$ They studied two populations, one of which concerned all quoted firms in manufacturing operating in the UK with 1957-assets in excess of $£$ 1 million. By carefully dissecting the sources of concentration growth, they conclude that, without mergers, (aggregate) concentration would not have increased much, and certainly not as much as it did during the 1957-1976 period they study. For the period 1957-1969, it was only the fact that the internal growth of large and merging firms was below the average of their population as a whole that prevented concentration from being higher than it already was. Interestingly, Hannah and Kay's study would suggest that, rather than attributing any departures from the Pareto curve to the effect of mergers, it is the influence of mergers that is to be held responsible for the Law of Proportionate Effect to hold, if it holds. First, if there were no mergers, the higher degree of diversification that is so characteristic of large firms would lead to a lower degree of dispersion of growth rates. Second, the feasibility

\footnotetext{
${ }^{4}$ Notice that the population involves large firms only.

${ }^{5}$ This assumes that there is no trade-off between internal and external growth, in the sense that it is assumed that firms would not have generated more internal growth if, for some reason, they had not focused on external growth.
} 
of an acquisition in terms of size will be dependent on the existing size of the acquiring firm. Merger, according to Hannah and Kay, is almost always the principal contributor to the variance of the growth of firms, since merger is behind most cases of outstandingly rapid growth.

Our contribution to the literature is twofold. First, this paper applies an extensive dataset of the entire population of Dutch manufacturing firms (and not only quoted or large firms), including entries and exits, to the study of the effects of M\&As on the shape of the firm size distribution. Second, it attempts to disentangle the effects of internal and external growth processes on the size distribution by means of a counterfactual analysis.

\section{Data and methodology}

We use two databases of manufacturing firms in the Netherlands collected by the Central Bureau of Statistics Netherlands (CBS). The first database is the Business Register (BR), a comprehensive database of the entire population of manufacturing firms. It contains demographic and domestic employment data of all firms registered for fiscal purposes in the Netherlands over the period 1993-1999. For a given year, the dataset includes all firms that had been active during that year, not necessarily for the full duration of that year. The set is composed of all firms that existed throughout the year and of all those that entered and/or exited during the year. Here, the events 'entry' and 'exit' are defined with respect to the inclusion in or exclusion from the dataset. In addition, the dataset specifies the reason for inclusion or exclusion of a firm. The particular variable allows distinguishing actual entry and exit of a firm from the entries and exits due to M\&As. Because of this identification and of the comprehensive scope of the dataset, (actual) entry and (actual) exit as reported in the BR provide very good approximations of the birth and death of a company. As a measure of size, we use the number of domestic employees, as this is the only measure available in the dataset. In this respect, the BR has the advantage of reporting the firm size down to zero employees (or self-employment).

The second database provides additional information beyond that in the BR. It contains detailed information on all domestic M\&As that took place within the observed population of firms from the BR. In particular, it allows matching the acquired (or merged) firms to the acquiring (or merging) firms, and the corresponding number of employees. From now on, we refer to an "event" whenever a merger, takeover or partial acquisition takes place, on the one hand; and, on the other, whenever restructuring of the company in different units, or via spin-offs and partial divestures takes place (partial acquisitions and divestitures are recorded only if they concern ownership changes beyond $50 \%$ of total outstanding shares).

Combining the two datasets, we have estimated two distributions: (a) the 'starting distribution' that one would observe at the beginning of the year, before any event had taken place; and (b) the 'final distribution' that one 
observes at the end of the year, after all events have taken place. The firms that carry out acquisitions (including partial acquisitions) or divestitures (including partial divestitures) appear in the starting distribution with their size before any event takes place. In the final distribution, they appear with their size modified according to the events that took place, i.e. in case of a merger or an acquisition with an increased size, while in case of a divestiture with a decreased size.

For a certain year, the 'starting distribution' is composed of four categories: (1) the firms that will not undergo any event throughout the year; (2) the firms that will exit during the year; (3) the firms that will be acquired or merged; and (4) the firms that will acquire or undertake partial divestiture. For the same year, the 'final distribution' comprises the following sets: (1) firms that will not have undergone any event during the year (so-called continuing firms); (2) firms that have entered during the year; (3) firms that have been spun-off or demerged from existing firms; and (4) firms that have done acquisitions or that have undertaken (partial) divestitures.

In addition, to distinguish better the effects of M\&A on the size distribution, we compare the starting and final state for the entire population-that is, as a result of (1) + (2) + (3) + (4) —as well as for the only set of firms involved in the process of M\&As-that is, (3) and (4).

As a reference year for the starting and final distributions, we consider the year 1997, since this is the year in which the greatest number of events related to M\&As took place over the observation period, 1993-1999. In 1997, the population of manufacturing firms from the Business Register includes 62,662 firms, of which slightly less than $10 \%$ are firms with zero employees (self-employment). Therefore, we compare the distribution that includes the firms with zero employees with the distribution that excludes these firms.

\section{The aggregate size distribution}

We first analyze the size distribution for the entire population of firms for 1997. We estimate the starting and final distributions for the population, including and excluding self-employment. Table 2 reports the descriptive statistics for the four distributions.

The number of active firms in the starting distribution amounts to 57,329, of which $9.7 \%$ are firms with zero employees (self-employment). The same number for the final distribution is equal to 56,595, of which 8.8 are selfemployed. A comparison of the starting and the final distribution shows that the number of firms decreases by $1.3 \%$ when self-employment is included, and with $0.2 \%$ when it is excluded. This suggests that the self-employed are suffering the highest mortality rate.

Our estimated distributions, no matter whether we are concerned with the starting or final distribution, either with or without self-employment, are in line with previous findings on empirical size distributions. Seventy-five percent of the distribution consists of firms with less that ten employees, confirming 
Table 2 Descriptive statistics of the starting and final distribution of the firm population in 1997

\begin{tabular}{|c|c|c|c|c|}
\hline & \multicolumn{2}{|c|}{ Including self-employment } & \multicolumn{2}{|c|}{ Excluding self-employment } \\
\hline & $\begin{array}{l}\text { Starting } \\
\text { distribution }\end{array}$ & $\begin{array}{l}\text { Final } \\
\text { distribution }\end{array}$ & $\begin{array}{l}\text { Starting } \\
\text { distribution }\end{array}$ & $\begin{array}{l}\text { Final } \\
\text { distribution }\end{array}$ \\
\hline Number of firms & 57,329 & 56,595 & 51,740 & 51,633 \\
\hline Mean number of employees & 16.9 & 17.0 & 18.7 & 18.6 \\
\hline SD & 185.5 & 181.8 & 195.1 & 190.2 \\
\hline Coff. variation & $1,096.5$ & $1,069.0$ & $1,041.3$ & $1,020.7$ \\
\hline Skewness & 137.2 & 137.6 & 130.5 & 131.6 \\
\hline Kurtosis & $25,789.3$ & $25,885.0$ & $23,312.0$ & $23,650.7$ \\
\hline \multicolumn{5}{|l|}{ Quantiles } \\
\hline $0 \%$ & 0 & 0 & 1 & 1 \\
\hline $1 \%$ & 0 & 0 & 1 & 1 \\
\hline $5 \%$ & 0 & 0 & 1 & 1 \\
\hline $10 \%$ & 1 & 1 & 1 & 1 \\
\hline $25 \%$ & 1 & 1 & 1 & 1 \\
\hline $50 \%$ & 2 & 2 & 3 & 2 \\
\hline $75 \%$ & 7 & 7 & 9 & 9 \\
\hline $90 \%$ & 25 & 25 & 27 & 27 \\
\hline $95 \%$ & 52 & 53 & 59 & 59 \\
\hline $99 \%$ & 238 & 245 & 261 & 267 \\
\hline
\end{tabular}

the "stylized fact" that size distributions are highly positively skewed: large numbers of small sized firms and small numbers of large firms. Indeed, only about $1 \%$ of the population has more than 230 employees. In addition, the mean of the distribution is between eight and nine times larger than the median, due to the long right tail (confirmed by the large kurtosis) given the presence of only a few large firms.

To compare graphically the size distributions of the starting and final populations, we plot the right cumulative distribution function on a double logarithmic scale (Fig. 2a) and the probability density function (Fig. 2b). Because of the logarithmic scale, we analyze only the distributions without self-employment. ${ }^{6}$

In Fig. 2a, b, the plots of the starting and final distribution largely overlap, thus demonstrating that the firm size distribution seems to be unaffected by events, among which are M\&As. Since 1997 was well into the fifth merger wave (see Fig. 1), and Dutch firms were among the world's most active in terms of M\&As, this would seem to be a puzzling result. However, a number of possible explanations can be brought forward. First, the dynamics of the entire manufacturing sector may counterbalance the effects of M\&As on the size distribution. In this case, we would assume that greenfield entrants $(8.13 \%$ of the population) as wells as the firms that exit the market $(7.09 \%$ of the population) offset the effects of M\&As on the size distributions.

\footnotetext{
${ }^{6}$ However, this restriction would not affect the analysis of the size distribution, since, as we observed in Table 2, the two distributions, with and without self-employment, have the same characteristic features.
} 
Fig. 2 Size distribution of all firms including M\&As in 1997. a Cumulative distribution function. b Probability density function

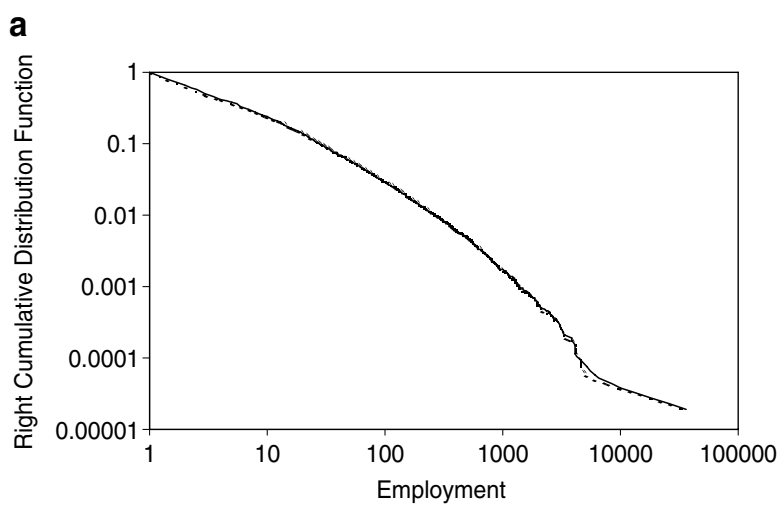

Starting distribution. ...... Final distribution

b

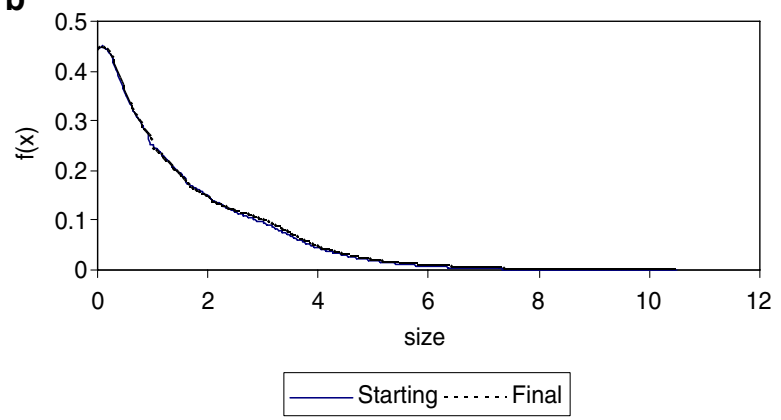

Second, our analysis focuses on the population of manufacturing firms that are active in the Netherlands, and on events related to M\&As that have involved these firms only, excluding events that took place abroad. In other words, we study the effects of M\&As on the firm size distribution as far as these firms have manufacturing facilities inside a country, in this case, the Netherlands. Especially during the last merger wave that ran from 19952000, many M\&As were cross-border, especially those involving large and very large firms. Dutch firms were particularly active in the international merger market. For example, in 1998, and in relation to home country gross domestic product (GDP), Dutch firms took the world's number one position in terms of cross-border M\&As. Such mergers amounted to approximately $11.1 \%$ of GDP, whereas cross-border M\&As involving French and German firms amounted to only $3.4 \%$ and 3.3\% of GDP, respectively (Schenk 2003). Therefore, even merger frenzies may leave domestic firm size distributionsas well as industrial concentration-unaffected, provided that the implied mergers are cross-border. Obviously, such mergers may have a significant effect on the firm size distribution as well as industrial concentration at the EU and/or the worldwide level. 
Finally, our analysis focuses on the effects of M\&As on the size distribution at the aggregate level, pooling together all manufacturing sectors. Thus, the result that no significant change of the firm size distribution has been detected may be due to aggregation. An increasing industrial concentration due to M\&As in one specific sector (at two or three digit level of the Standard Industrial Classification) might be compensated by a decreasing concentration in another. Therefore, aggregation could have washed away the effects of M\&As on industrial concentration.

In order to test whether the differences between the two distributions are statistically significant, we run a Kolmogorov-Smirnov test. The results shown in Table 3 allow us to reject the hypothesis that the two empirical distributions come from the same theoretical distribution. Despite the graphical analysis, the statistical test detects the effects of the M\&As on the size distribution. Indeed, we know that about 3,000 firms are involved in such processes. As the Kolmogorov-Smirnov is a sensitive test for a large number of observations, it is able to capture an effect that the graphical analysis neglects.

We calculate the Herfindahl index for the starting and final distributions in order to assess whether the degree of industrial concentration changes as an effect of industrial dynamics. Defining $N$ the number of the firms in the distribution (the industry of interest), $s_{i}$ the size of firm $i$ and $f_{i}$ the market share of firm $i$, the Herfindahl index is given by

$$
H=\sum_{i=1}^{N} f_{i}^{2}
$$

where

$$
f_{i}=\frac{s_{i}}{\sum_{i=1}^{N} s_{i}}
$$

The index ranges from the $\operatorname{Min}(H)=1 / N$ to the $\operatorname{Max}(H)=1$. Therefore, the minimum value of the index depends on the number of the firms in the distribution.

In order to avoid such shortcoming and to enable comparisons between the indexes calculated on different distributions with different numbers of

Table 3 Kolmogorov-Smirnov two-sample test (asymptotic)

\begin{tabular}{lllllll}
\hline & $\begin{array}{l}\text { Starting } \\
\text { distribution } \\
(\mathrm{N})\end{array}$ & $\begin{array}{l}\text { Final } \\
\text { distribution } \\
(\mathrm{N})\end{array}$ & KS & D & KSa & Pr $>$ KSa \\
\hline $\begin{array}{c}\text { All firms: including } \\
\text { those with zero employees }\end{array}$ & $57,329.00$ & $56,595.00$ & 0.00 & 0.01 & 1.66 & 0.01 \\
$\begin{array}{c}\text { All firms: excluding those } \\
\text { with zero employees }\end{array}$ & $51,740.00$ & $51,633.00$ & 0.01 & 0.02 & 2.44 & $<.0001$ \\
$\begin{array}{c}\text { Only firms involved in M\&A: } \\
\text { including those with } \\
\text { zero employees }\end{array}$ & $3,899.00$ & $2,564.00$ & 0.13 & 0.26 & 10.17 & $<.0001$ \\
\hline
\end{tabular}


observations, we calculate the relative measure of concentration $H^{*}$ that normalizes for the number of firms in the distribution. Namely,

$$
H^{*}=\frac{H-\operatorname{Min}(H)}{\operatorname{Max}(H)-\operatorname{Min}(H)}=\frac{H-1 / N}{1-1 / N}
$$

with $\operatorname{Min}\left(H^{*}\right)=0$ and $\operatorname{Max}\left(H^{*}\right)=1$

It holds that:

$$
H^{*}=\frac{N^{2}}{N-1} V
$$

where $V$ is the variance of firms market shares

$$
V=\frac{\sum\left(f_{i}-1 / N\right)^{2}}{N}
$$

For the starting distribution, the normalized Herfindahl index is 0.002115 and for the final distribution 0.002037. The result suggests that, in 1997, the industrial dynamics slightly reduced the degree of the domestic industrial concentration.

This counterintuitive result could be explained by observing that the increase in the density of the medium-sized firms outweighs the increase in the density of the upper tails of the distribution. Taking into account Eq. 2, we can notice that, as the number of firms in the distribution decreases (as happened from the starting to the final distribution in 1997, that is, from 57,329 to 56,595) and keeping constant the variance of the distribution, the value of the normalized Herfindahl index decreases. In order to have an increase of the Herfindahl index, we should observe an increase of the variance of the distribution that offsets the decrease due to the reduction in the number of firms. This is what usually happens when the majority of the M\&As takes place among large firms, giving rise to even larger or gigantic firms. In our case, instead, we consider what happens to the distribution of the entire population of firms due to the M\&As events among all classes. What we observe in our data is that the density of the distribution significantly increases around the mean of the distribution, and the variance of the distribution decreases for the effects of the M\&As (from $3.7 \times 10^{-9}$ to $3.6 \times 10^{-9}$ ), enhancing the effects of the reduction of the number of firms. As a result, the Herfindahl index decreases.

Finally, to investigate the shape of the size distribution, and the effects of industrial dynamics, we present the p-p plots for the starting and final distributions both for the lognormal and Pareto. Figure 3 shows that the lognormal distribution fits the data better than the Pareto distribution. This is not surprising because we know from the literature that the Pareto distribution fits better the upper tail of the firm size distribution (Marsili 2005; Cabral and Mata 2003). As observed for the density and cumulative distribution graphs, the differences in the shape between the starting and the final distributions cannot be visually appreciated. 
Lognormal P-P Plot of Starting distribution

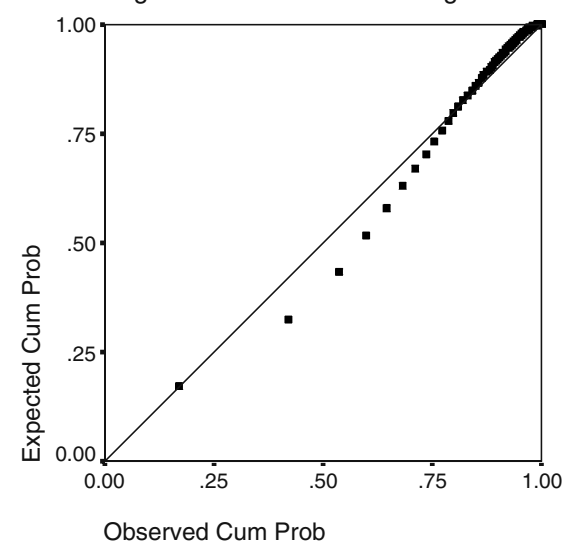

Lognormal P-P Plot of Final distribution

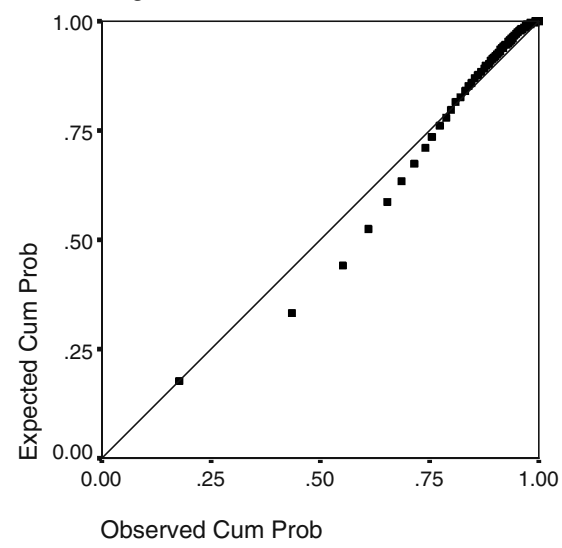

Pareto P-P Plot of Starting distribution

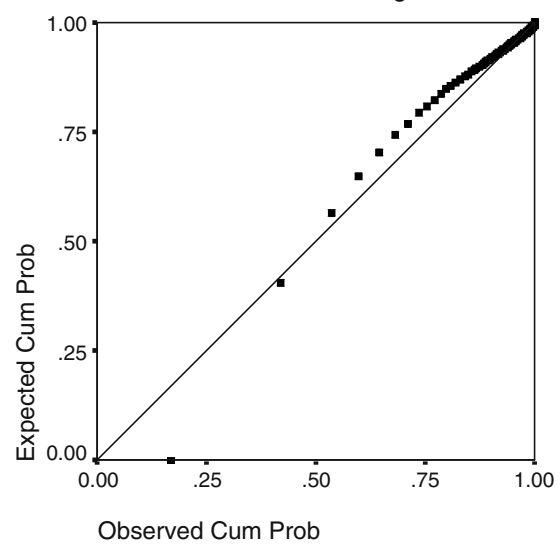

Pareto P-P Plot of Final distribution

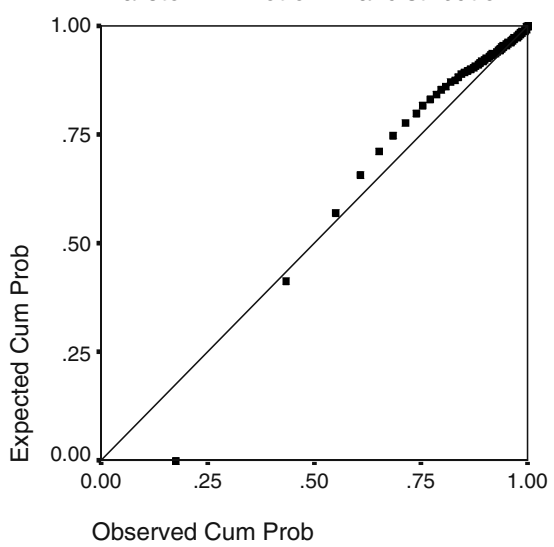

Fig. 3 Lognormal (left) and Pareto (right) p-p plots for the starting and final distributions of firm size (entire population)

\section{The effects of mergers and acquisitions}

In this section, we focus on the set of firms that are involved in an M\&A event during the year 1997. In this way, we try to emphasize the possible effects of M\&As on the shape of the size distribution. The starting distribution represents the initial (at the beginning of the year) size of the firms that we know will be involved in an event during the year. The final distribution collects the size of the same firms after the event took place. Furthermore, in the final distribution, the new firms created by spin-offs, divestures and restructuring appear, while firms that exited the market due to mergers or takeovers are no longer present. 
During 1997, 3,899 firms were involved in activities related to M\&As and their spin-offs and divestures. At the end of the year, 2,564 firms remained, which suggests that M\&As, spin-offs, and divestitures have decreased the numbers of firms active in the manufacturing sector. The descriptive statistics shown in Table 4 highlight that this is not the only effect. The average firm size increases by $56 \%$, while the median increases by $100 \%$. Looking at the quintiles of the two distributions (the one at the beginning of the year and the one at the end, after all events had occurred), one could conclude that the entire starting distribution shifts towards larger sizes

It is worth noting that the skewness, the kurtosis and also the coefficient of variation considerably decrease from the starting to the final distribution, suggesting that the final distribution is more symmetric and more concentrated around its mean.

As Fig. 4 confirms, there are more firms concentrated in the central part of the distribution. Indeed, the density increases drastically at the center, and marginally in the upper tail. This increase is due to a large reduction in the density of firms in the lower tail (see Fig. 4b). Overall, we observe that the effect of M\&As increases significantly the number of medium-sized firms, reducing the number of the micro and small firms, while the number of large firms does not increase in such a relevant way, but their size increases considerably (see the quantiles at $95 \%$ and $99 \%$ in Table 4 : the values almost double).

In accordance with the graphical analysis, the Kolmogorov-Smirnov test in Table 3 shows that the empirical distributions before and after the M\&A events come from two distinct theoretical distributions.

Table 4 Descriptive statistics of the number of employees of the firms involved in events during 1997

\begin{tabular}{lcc}
\hline & \multicolumn{2}{c}{ Including those with zero employees } \\
\cline { 2 - 3 } & Starting distribution & Final distribution \\
\hline Number of firms & 3,899 & 2,564 \\
Mean & 36.33 & 55.67 \\
SD & 621.16 & 748.85 \\
Coff. variation & $1,709.70$ & $1,345.23$ \\
Skewness & 52.48 & 42.54 \\
Kurtosis & $3,003.15$ & $1,973.80$ \\
Quantiles & & \\
$0 \%$ & 0 & 0 \\
$1 \%$ & 0 & 0 \\
$5 \%$ & 0 & 2 \\
$10 \%$ & 0 & 4 \\
$25 \%$ & 2 & 8 \\
$50 \%$ & 7 & 14 \\
$75 \%$ & 15 & 21 \\
$90 \%$ & 27 & 45 \\
$95 \%$ & 64 & 122 \\
$99 \%$ & 350 & 579 \\
\hline
\end{tabular}


Fig. 4 Size distribution of firms involved in M\&As in 1997. a Cumulative distribution function. b Probability density function

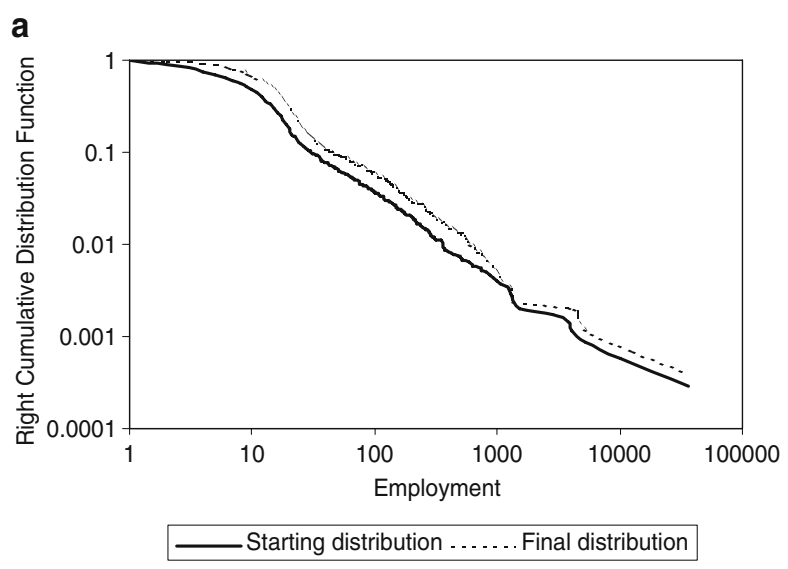

b

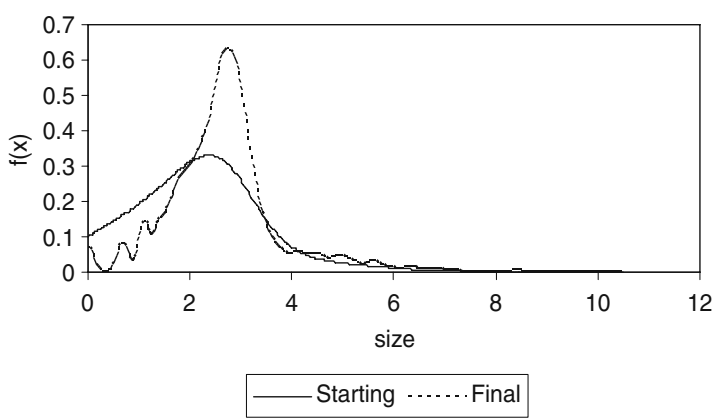

As before, we calculate the Herfindahl index of the starting and final distributions in order to measure the changes in industrial concentration due to the effects of M\&As. For the starting distribution, the Herfindahl index is 0.07673 , while it is 0.07207 for the final distribution. ${ }^{7}$

As with for the entire population, industrial concentration decreases as an effect of M\&As but for a different composition of effects with respect to the entire population. The density of the distribution significantly increases around the mean of the distribution, but, in this case, the variance of the distribution increases for the effects of the M\&As (from $2.0 \times 10^{-6}$ to $2.8 \times 10^{-6}$ ), but not enough to offset the effects of the reduction of the number of firms. As a result, also in this case, the Herfindahl index decreases. This is consistent with the decline of the coefficient of variation from the starting distribution to the final distribution, both in the entire population and in the sample considering only firms involved in M\&A events.

Another way to study the effects of M\&As on the shape of the size distribution is to look at the $\mathrm{p}-\mathrm{p}$ plots. Figure 5 reports the $\mathrm{p}-\mathrm{p}$ plots for the

\footnotetext{
${ }^{7}$ As before, the measure of concentration has been calculated using a relative measure that normalizes for the number of firms in the distribution.
} 
Lognormal P-P Plot of Starting distribution

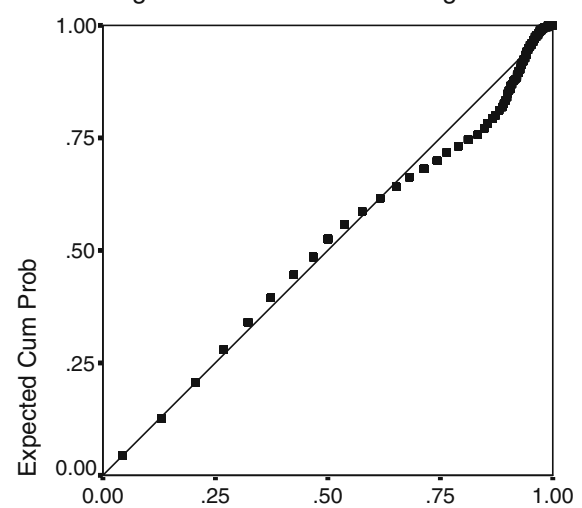

Observed Cum Prob

Lognormal P-P Plot of Final distribution

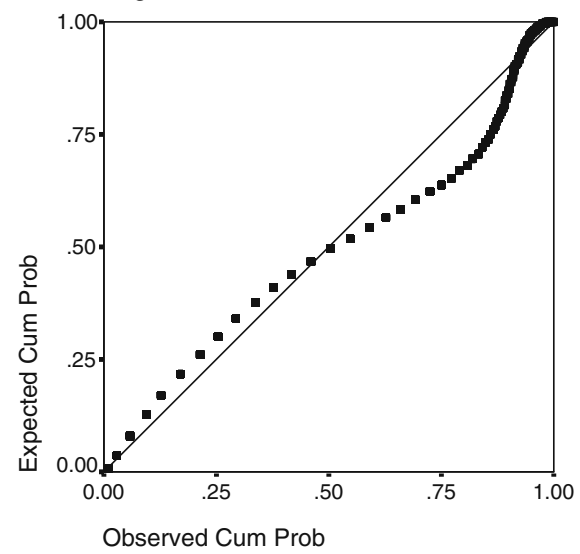

Pareto P-P Plot of Starting distribution

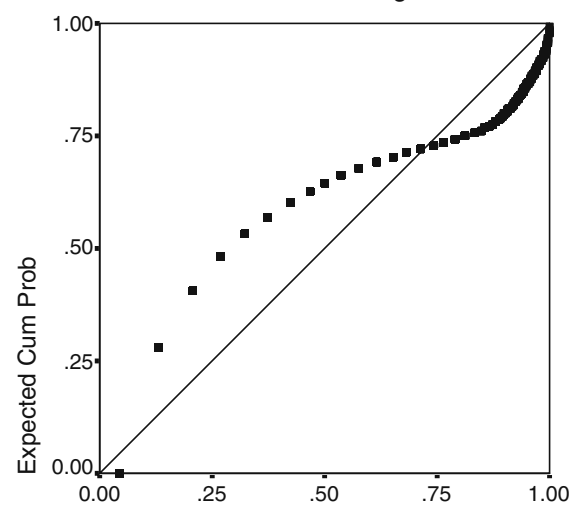

Observed Cum Prob

Pareto P-P Plot of Final distribution

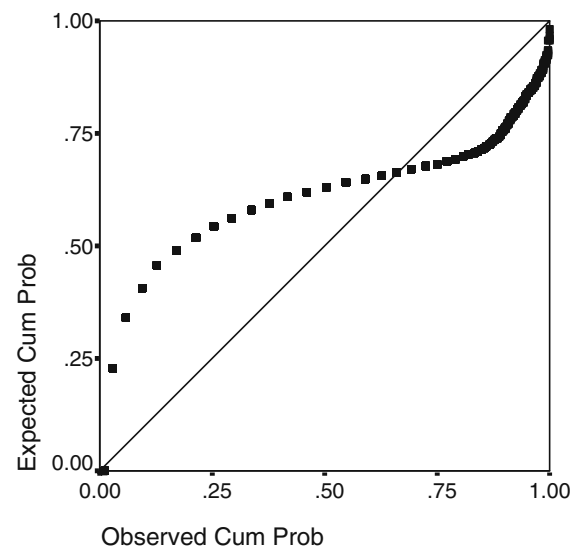

Fig. 5 Lognormal (left) and Pareto (right) p-p plots for the starting and final size distributions for only firms involved in M\&A

lognormal and Pareto. Again, the Pareto shows a poor fit to the data, while the lognormal seems to provide a better fit. If we focus on the lognormal $\mathrm{p}-\mathrm{p}$ plot of the final distribution, we can observe that the M\&As induce a greater departure from log-normality than the starting distribution. This suggests that the process of external growth due to M\&As is not generated by Gibrat's process.

\section{Counterfactual analysis}

The differences previously observed between the starting and the final distributions reflect organic or internal growth of the firm as well as growth due to M\&As (external growth). In order to disentangle the effects given by 
internal and external growth, we perform a counterfactual analysis. The aim is to compare the real final distribution of firms that underwent an M\&A event (including any internal growth as well as external growth) with the distribution of the same firms, assuming that they experienced only internal growth.

The computation of the counterfactual distribution builds on the following steps. First, the actual growth rates between the years 1997 and 1998 for the firms that are not involved in an M\&A event are calculated. From this distribution of actual growth rates, a random sample was extracted of dimension equal to the number of firms involved in M\&A. By applying these random values to the initial size of firms involved in an M\&A event, we derive the final size of these same firms, had they not been involved in M\&As. The actual final size of firms involved in M\&A and the imputed final size of those that were involved in M\&A define the final distribution of firm size that would result from internal growth only.

The counterfactual analysis (see Fig. 6a) suggests that M\&As do affect the firm size distribution, especially among medium sized firms: indeed, the density of the M\&A-firms is much above the counterfactual in the central part of the distribution, meaning that M\&As create a large number of medium sized firms. The upper tail is less affected by M\&As than is the lower tail. The number of large firms slightly rises, but, above all, the size of very few large firms increases as an effect of M\&A. In fact, Fig. 6a shows a longer and slightly thicker upper tail of the final distribution than the counterfactual distribution. However, the dynamics that we observe among the largest firms is limited in number in

Fig. 6 Counterfactual analysis. a Real final distribution versus the counterfactual distribution (only firms involved in M\&As). b The effects of only internal growth: starting versus counterfactual distribution
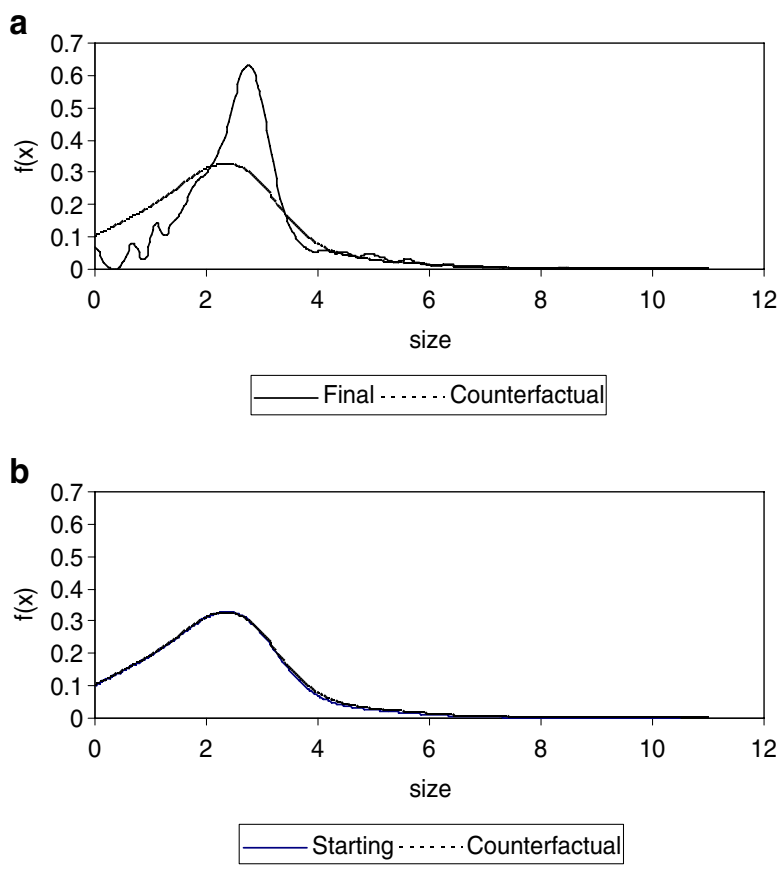
comparison to the dynamics of the small and medium sized firms. The total effect of M\&As is that industrial concentration, as measured by the normalized Herfindahl index, is higher in the counterfactual distributions $(H=0.0965)$ than in the actual final distribution $(H=0.07027)$. This is mainly because, in the final distribution, the number of firms with size around the mean increases considerably at the expense of micro and small firms. This variation largely outweighs the increase in number and average size of very few large firms and makes the concentration index decrease.

Figure $6 \mathrm{~b}$ shows that only internal growth does not affect the shape of the size distribution of firms. Indeed, the starting and counterfactual distributions are hardly distinguishable in the graph. On the contrary, the counterfactual analysis suggests that the change in the size distribution is almost entirely due to the external growth of the firms.

\section{Conclusions}

As pointed out by Scherer (2002), the process of M\&As plays an important role in shaping market concentration. Nevertheless, the empirical evidence on how this process influences the shape of the firm size distribution is limited and somewhat inconclusive. This paper provides new empirical evidence on the effects of M\&As on the shape of the size distribution of firms, by using data from the Business Register of the population of manufacturing firms in the Netherlands.

Our analysis shows that M\&As do not affect the size distribution when we consider the entire population of firms. This may depend on a number of facts: that entries and exits may balance the effects of M\&As, that the distribution may aggregate opposite effects at the sector level, and that international M\&As, which are excluded from the dataset, may have the most apparent effect on the overall population because they tend to involve the largest firms.

The effects of M\&A on the shape of the size distribution emerge when we focus on the firms that were involved in a merger or acquisition event in the observed period. First of all, we observed a shift of the firm size distribution towards larger sizes, with a considerable increase in both the mean and median of firm size. At first glance, this is consistent with what Ijiri and Simon (1971) originally noted in their study of US largest firms in the 1950s and early 1960s. They found that M\&As produced an upward shift in the Pareto curve, which, however, left the shape of the distribution-and therefore market concentration as measured by the slope of the Pareto curve-largely unchanged. Ijiri and Simon's interpretation was that the size distribution was relatively invariant to the process of M\&As, because all firms grew roughly in the same proportion as an it.

Our analysis of the higher central moments of the distribution allowed us to qualify such a shift in firm size and provided evidence that the shape of the distribution did change per effect of M\&As (in contrast with the results 
of the 1971 study by Ijiri and Simon). Indeed, we found that the firm size distribution becomes more concentrated around the mean, less skewed to the right hand side, and thinner at the tails as a whole. In addition, the graphical inspection of the density functions revealed that the shift toward higher sizes due to M\&A is not uniform but affects firms of different sizes in different ways. While the number of firms in the lower tail decreased, as expected, the number of firms in the central size classes increased substantially and outweighed the increase in the number (and mean size) of firms in the upper tail of the distribution.

In sum, we observe increasing concentration of firms around the average size, which seems to counterbalance the observed increase in firm size in the upper tail of the size distribution as an effect of M\&As (consequently the overall market concentration measured by the normalized Herfindahl index declines). These findings are consistent with the results of a later work by Ijiri and Simon (1974) in which they revised their earlier conclusions, on the basis of data from the late 1960s; during this period a larger number of M\&As took place than in the 1950s and early 1960s. Ijiri and Simon (1974) observed an increase in concavity in size distribution-compared to the straight line of the Pareto law-when the number of M\&As picked up. The increase in concavity implies that the number of firms in the center of the distribution increases, which is consistent with our results.

Another aspect is the implications on Gibrat's law. Firms disappear both at the bottom of the distribution and somewhere in the middle-high range. The probability of experiencing an M\&A is not uniform over the size range. This process leads to a change in the shape of the size distribution and to a departure from log-normality. The departure from the lognormal is more evident for the firms involved in M\&As than for the total population. This suggests that external growth does not follow Gibrat's law, and even more so than in the overall population. Small firms are more likely to be acquired, while the larger are more likely to merge (Singh 1975).

Our counterfactual analysis highlights the idea that only internal growth does not affect the shape of the size distribution of firms. On the contrary, it suggests that the change in the size distribution is almost entirely due to the external growth of the firms. More research is needed here, on the relationship between firm growth rates and different form of growth, internal and external. Our analysis is limited to the effects that M\&As in a certain year had on the firm size distribution in aggregate manufacturing. As possible extensions of the current research, it would be interesting to see whether the effects of M\&As that we observed are invariant or not over time and across industrial sectors. One could then examine the evolution of the effects of M\&As on the firm size distribution over time at the economy wide level and at the disaggregated level of sectors.

Acknowledgements The authors would like to thank the participants in the 10th International Schumpeter Society Conference held in Milan in 2004, and in particular G. Bottazzi, S. Klepper, J. Sutton and A. Secchi for helpful and interesting suggestions. The empirical part of this research 
has been carried out at the Centre for Research of Economic Microdata, at Statistics Netherlands. The views expressed in this paper are those of the authors and do not necessarily reflect the policies of Statistics Netherlands. The authors gratefully acknowledge the financial support of NWO (Dynamics of Innovation Programme, grant nr: 472-04-008). Elena Cefis also acknowledges the financial support of the University of Bergamo (grant ex 60\%, n. 60CEFI07, Dept. of Economics).

Open Access This article is distributed under the terms of the Creative Commons Attribution Noncommercial License which permits any noncommercial use, distribution, and reproduction in any medium, provided the original author(s) and source are credited.

\section{References}

Aaronovitch S, Sawyer M (1975) Big business. Macmillan, London

Blair JM (1972) Economic concentration. Harcourt Brace, Jovanovich

Bottazzi G, Secchi A (2006) Explaining the distribution of firm growth rates. RAND J Econ 37(2):235-256

Bottazzi G, Cefis E, Dosi G (2002) Corporate growth and industrial structure. Some evidence from the Italian manufacturing industry. Ind Corp Change 11(4):705-725

Cabral L, Mata J (2003) On the evolution of the firm size distribution: facts and theory. Am Econ Rev 93(4):1075-1090

Cefis E, Ciccarelli M, Orsenigo L (2007) Testing Gibrat's legacy: a Bayesian approach to study the growth of firms. Struct Chang Econ Dyn 18(3):348-369

Geroski PA (1999) The growth of firms in theory and in practice. CEPR working paper 2092. CEPR, London

Gibrat R (1931) Les Inegalites economiques. Sirey, Paris

Hannah L, Kay JA (1977) Concentration in modern industry. Macmillan, London

Hannah L, Kay JA (1981) The contribution of mergers to concentration growth: a reply to professor Hart. J Ind Econ 29(3):305-313

Ijiri Y, Simon HA (1971) Effects of mergers and acquisitions on business firm concentration. J Polit Econ 79(2):314-322

Ijiri Y, Simon HA (1974) Interpretations of departures from the Pareto curve firm-size distributions. J Polit Econ 82(2):315-331

Krugman PR (1996) The self-organizing economy. Blackwell, Cambridge

Lotti F, Santarelli E (2004) Industry dynamics and the distribution of firm sizes: a nonparametric approach. South Econ J 70(3):443-466

Luttmer EGJ (2007) Selection, growth, and the size distribution of firms. Q J Econ 122(3): 1103-1144

Marsili O (2005) Technology and the size distribution of firms: evidence from Dutch manufacturing. Rev Ind Org 27(4):1-26

McCloughan P (1995) Simulation of concentration development from modified Gibrat Growthentry-exit processes. J Ind Econ 43(4):405-433

Odagiri H (1992) Growth through competition, competition through growth. Oxford University Press/Clarendon, Oxford

Pryor FL (2001) Will most of us be working for giant enterprises by 2028? J Econ Behav Organ 44:363-382

Schenk H (1997) Mergers, efficient choice, and international competitiveness. Bandwagon behaviour and industrial policy implications, working paper. Erasmus University Rotterdam, Rotterdam

Schenk H (2003) Organisational economics in an age of restructuring, Inaugural Address. Utrecht University, Ultrecht

Scherer FM (2002) Assessing progress in research on industrial evolution and economic growth: a review article. Small Bus Econ 16:237-242

Singh A (1975) Take-overs, economic natural selection and the theory of the firm: evidence from the post-war United Kingdom experience. Econ J 85(339):497-515

Sutton J (1997) Gibrat's legacy. J Econ Lit 35:40-59, March 